\title{
Resting State Functional Connectivity Signature of Treatment Effects of Repetitive Transcranial Magnetic Stimulation in Mal de Debarquement Syndrome
}

\author{
Han Yuan,,2 Guofa Shou, Diamond Gleghorn, Lei Ding,, ${ }^{1,2}$ and Yoon-Hee Cha ${ }^{2}$
}

\begin{abstract}
Repetitive transcranial magnetic stimulation (rTMS) has been used in experimental protocols to treat mal de debarquement syndrome (MdDS), a neurological condition that represents a maladaptive brain state resulting from entrainment to external oscillating motion. Medical treatments and biomarkers for MdDS remain limited but neuromodulation with rTMS has shown evidence for therapeutic effects. This study took a neuroimaging approach to examine the neuromodulatory effect of rTMS on MdDS. Twenty individuals with MdDS underwent five daily treatments of rTMS over bilateral dorsolateral prefrontal cortex (DLPFC). Participants received $1 \mathrm{~Hz}$ over right DLPFC (1200 pulses) followed by $10 \mathrm{~Hz}$ over left DLPFC (2000 pulses). Resting state functional magnetic resonance imaging was acquired before and after treatments to determine functional connectivity changes associated with a positive treatment effect. A single-subject-based analysis protocol was developed to capture the degree of resting state functional connectivity (RSFC) between the rTMS target and the entorhinal cortex (EC), an area previously shown to be hypermetabolic in MdDS. Our results showed that rocking motion perception in subjects was modulated by rTMS over the DLPFC. Improvements in symptoms correlated most strongly with a post-rTMS reduction in functional connectivity between the left EC and the precuneus, right inferior parietal lobule, and the contralateral EC, which are part of the posterior default mode network. Positive response to rTMS correlated with higher baseline RSFC between the DLPFC and the EC. Our findings suggest that baseline prefrontal-limbic functional connectivity may serve as a predictor of treatment response to prefrontal stimulation in MdDS and that RSFC may serve as a dynamic biomarker of symptom status.
\end{abstract}

Keywords: BOLD fMRI; dorsolateral prefrontal cortex; resting state functional connectivity; transcranial magnetic stimulation

\section{Introduction}

$\mathbf{E}$ XPOSURE TO PROLONGED periods of low frequency low amplitude oscillating motion can trigger postmotion feelings of rocking and swaying, lasting very briefly in the majority of individuals but persisting for months or years in an important minority (Brown and Baloh, 1987; Gordon et al., 1995). This phenomenon, called mal de debarquement syndrome (MdDS), has been recognized for centuries (Irwin, 1881), but only recently have neuroimaging studies shown metabolic, brain volume, and functional connectivity changes related to this disorder (Cha, 2012; Cha and Chakra- pani, 2015). The conditions that promote the development of MdDS appear to be related to age, gender, stress during travel, dynamic changes in hormone levels, and perhaps certain medications (Cha, 2015; Cha et al., 2008; Hain et al., 1999). Treatment options for MdDS remain severely limited, especially for symptoms lasting more than a year that have an extremely low chance for remission (Cha, 2015). This motivates the exploration of new therapies.

Standard clinical structural brain imaging and vestibular function testing are normal in MdDS. However, a previous functional imaging study with fluorodeoxyglucose positron emission tomography showed that the left entorhinal cortex

\footnotetext{
${ }^{1}$ Stephenson School of Biomedical Engineering, University of Oklahoma, Norman, Oklahoma.

${ }^{2}$ Laureate Institute for Brain Research, Tulsa, Oklahoma.

(C) Han Yuan et al. 2017; Published by Mary Ann Liebert, Inc. This is an Open Access article distributed under the terms of the Creative Commons Attribution License, which permits unrestricted use, distribution, and reproduction in any medium, provided the original work is properly cited.
} 
(IEC)-amygdala junction is hypermetabolic in MdDS, which is significant because this region has widespread connectivity with large portions of the neocortex, receiving sensory input from posterior parietal and occipital cortices and being regulated by the prefrontal cortex (Cha, 2012; Coutureau and Di Scalam, 2009; Dickson et al., 2000; Van Hoesen et al., 1972). A theory for the cause for MdDS has been developed that the entorhinal cortex (EC) may be tuned by external neural input; entrainment to persistent oscillating sensory input through vestibular and somatosensory cortices may change the latent activity of the EC (Jacob et al., 2014; Kajiwara et al., 2003). EC neurons have previously been shown to exhibit graded persistent activity that changes in a step-wise rather than continuous manner (Egorov et al., 2002). This suggests that sensory inputs to the EC might allow it to switch, like a toggle, between higher and lower states of synchronization (Dupret and Csicsvari, 2012; Hasselmo et al., 2000; Tahvildari et al., 2007). This is consistent with the clinical behavior of the disorder in which symptoms can quickly shut off over the course of a day but then come back on as quickly. We hypothesized that such synchronization might be directly manipulated by inducing external oscillating input in the form of transcranial magnetic stimulation (TMS), and that the manipulation of neuronal synchronization may correlate with symptom status.

Repetitive TMS (rTMS) has proven to be effective in treating various neurological and neuropsychiatric conditions (Hallett, 2007). TMS induces electrical currents in cortical tissue targeted by the configuration of the stimulation coils. Pulses of stimulation can modulate cortical excitability using either inhibitory low-frequency $(\leq 1 \mathrm{~Hz})$ or facilitatory high-frequency stimulation $(\geq 5 \mathrm{~Hz}$ ) (Di Lazzaro et al., 2011; Hallett, 2007; Noh et al., 2012). The response to stimulation can be observed not only in local stimulated areas but also in remote sites that are anatomically and/or functionally connected. Moreover, rTMS has proven to be effective in inducing lasting therapeutic effects in disease conditions. One of the first Food and Drug Administration-approved therapeutic indications for rTMS was high-frequency stimulation to the left dorsolateral prefrontal cortex (IDLPFC) for the treatment of medication-resistant depression. It has been hypothesized that dorsolateral prefrontal cortex (DLPFC) rTMS might have distributed effects on deeper limbic regions (George et al., 1995, 1997; Padberg and George, 2009), although combined TMS and neuroimaging studies designed to investigate this hypothesis have produced conflicting results regarding the nature of such effects (George et al., 1999; Knoch et al., 2006; Li et al., 2004; Speer et al., 2000).

Using high-density electroencephalograms (EEGs) and an algorithm developed to quantify electrophysiological functional connectivity with independent component analyses, Ding and associates showed that rTMS of the DLPFC in individuals with MdDS led to symptom improvement, correlating with a reduction in a measure of synchrony called the independent component phase coherence (ICPC) (Ding et al., 2014; Shou et al., 2014). ICPC is a measure of intrinsic functional connectivity calculated within specific frequency bands in EEG data and is a marker of the degree of synchronization between brain regions. Individuals with MdDS who improved with rTMS showed a reduction in global coherence as well as a decrease in ICPC between specific nodes within posterior parietal and occipital lobes (Ding et al., 2014). This was consistent with the theory of the acquisition of MdDS that exposure to oscillating motion sets up a process of abnormal oversynchronization and that a return to a healthy brain state involves reducing abnormal functional connectivity (Cha, 2015). However, it remains unknown whether or how rTMS manipulates the connectivity with maladaptive limbic regions that have been previously shown to be hypermetabolic (Cha, 2012).

Functional magnetic resonance imaging (fMRI) of the brain during rest reveals large-amplitude spontaneous lowfrequency $(<0.1 \mathrm{~Hz})$ fluctuations that are temporally correlated across functionally related areas (Biswal et al., 1995). These correlations, referred to as resting state functional connectivity (RSFC), yield detailed maps of complex neural systems (Biswal et al., 2010). RSFC has also been suggested as sensitive measurements to reflect neuromodulatory effects (Fox et al., 2012a; Yuan et al., 2014). Thus, the rationale for this study was to determine whether functional connectivity with the EC increases or decreases as a function of symptom improvement after brain stimulation in MdDS. Second, if these functional connectivity measures are relevant to symptom change, there may also be baseline functional connectivity markers that might predict response to brain stimulation. This study builds on prior work using rTMS to induce symptom changes in MdDS by targeting DLPFC (Cha et al., 2013, 2016; Ding et al., 2014) but also includes pre- and post-rTMS functional imaging to assess RSFC changes as a result of treatment response.

\section{Materials and Methods}

\section{Informed consent}

Study procedures were completed according to Declaration of Helsinki guidelines and approved through Western IRB (www.wirb.com). Participants provided written informed consent and were recruited under ClinicalTrials.gov study NCT02470377. The data presented here is limited to the imaging data analyses as details of the clinical data were previously reported in Cha and associates (2016). This study used rTMS in an off-label manner.

\section{Inclusion and exclusion criteria}

Criteria for inclusion were the following: (1) chronic perception of rocking vertigo that started within 2 days after disembarking from water, air, or land-based travel, (2) symptoms lasting at least 6 months, (3) no other cause for symptoms after evaluation by a neurologist or otolaryngologist with appropriate testing for peripheral inner ear or other central nervous system causes for symptoms. Exclusion criteria include the following: (1) an unstable medical or psychiatric condition, including a history of bipolar disorder or psychosis, (2) pregnant or planning to become pregnant during the course of the study, (3) contraindications to receiving rTMS or MRI, including medications known to reduce seizure threshold, (4) an unclear history of the onset of symptoms, and (5) an inability to complete all study-related testing.

\section{Participant characteristics}

Twenty right-handed individuals with MdDS started and completed all 5 days of the protocol. Although the study was open to either gender, only female participants 
volunteered for the study. This was consistent with the much higher female than male prevalence in MdDS (Cha, 2012). The mean age at the time of the study was $52.9 \pm 12.6$ years, median of 56.5 years, and a range of 28-68 years. The duration of illness had a mean of $35.2 \pm 24.2$ months, median 30 months, and a range of 8-96 months. Motion exposures preceding the onset of MdDS included ocean/sea travel in 11, air travel in 4, and land-based (car or train) travel in 5.

\section{Magnetic resonance imaging}

Functional and structural magnetic resonance images were collected using a General Electric Discovery MR750 wholebody 3-Tesla MRI scanner (GE Healthcare, Milwaukee, WI). A brain-dedicated receive-only 32-element coil array (Nova Medical, Inc., Wilmington, MA), optimized for parallel imaging, was used for MRI signal reception. A single-shot gradient-recalled echo-planar imaging (EPI) sequence with sensitivity encoding depicting blood-oxygenation-level-dependent (BOLD) contrast was used for functional scans. The following EPI parameters were used: field of view/slice/gap $=220$ / $3.0 / 0 \mathrm{~mm}$, axial slices per volume $=42$, acquisition matrix $=$ $64 \times 64$, repetition/echo time $=2000 / 30 \mathrm{msec}$, acceleration factor $\mathrm{R}=2$ in the phase encoding (anterior-posterior) direction, flip angle $=90^{\circ}$, sampling bandwidth $=250 \mathrm{kHz}$, number of volumes $=120$, and scan time $=240 \mathrm{sec}$. All EPI images were reconstructed into a $128 \times 128$ matrix, in which the resulting fMRI voxel volume was $1.7 \times 1.7 \times 2.9 \mathrm{~mm}^{3}$. In addition, simultaneous physiological pulse oximetry and respiration waveform recordings were conducted (with $40 \mathrm{~Hz}$ sampling) for each fMRI run. A photoplethysmograph with an infrared emitter placed under the pad of the subject's left index finger was used for pulse oximetry, and a pneumatic respiration belt was used for respiration measurements. A T1-weighted magnetization-prepared rapid gradient-echo sequence with sensitivity encoding was used to provide an anatomical reference for the fMRI analysis and had the following parameters: field of view $=240 \mathrm{~mm}$, axial slices per slab $=190$, slice thickness $=0.9 \mathrm{~mm}$, image matrix $=256 \times 256$, repetition time/echo time $=5 / 2.012 \mathrm{msec}$, acceleration factor $\mathrm{R}=2$, flip angle $=8^{\circ}$, inversion time TI $=725 \mathrm{msec}$, and sampling bandwidth $=31.2 \mathrm{kHz}$. Day 1 pre-TMS and day 5 post-TMS imaging sessions were performed at the same time of the day, including care taken to acquire imaging data at the same time interval from the last meal.

\section{Repetitive transcranial magnetic stimulation}

Each participant underwent five sessions of rTMS on consecutive days, typically at the same time each day. The Localite TMS Navigator (Localite GmBH, Germany) frameless stereotaxy system was used for neuronavigation to identify the center of the DLPFC as the anterior portion of the middle of the middle frontal gyrus. rTMS was performed with the Magventure MagPro X100 stimulator with a cooled figure-of-eight coil in biphasic mode and the handle back at a $45^{\circ}$ angle relative to the midsagittal plane. Motor thresholds (MTs) were determined each day with independent measurements made for the hand areas in both right and left primary motor cortices before each treatment session. Each motor hand area (the "hand knob") was identified with subjectspecific neuronavigation. MTs were defined as the percentage intensity of the stimulator output that generated a
$50 \mu \mathrm{V}$ motor evoked potential in the contralateral abductor pollicis brevis muscle in 5 out of 10 trials. rTMS sessions consisted of $1 \mathrm{~Hz}$ right DLPFC (rDLPFC) stimulation at $110 \%$ of MT for 1200 pulses followed by $10 \mathrm{~Hz}$ IDLPFC stimulation at $110 \%$ MT for 2000 pulses. The right MT was used for the rDLPFC treatment and the left MT was used for the IDLPFC treatment. The $10 \mathrm{~Hz}$ protocol was administered as trains of 40 pulses over $4 \mathrm{sec}$ followed by $26 \mathrm{sec}$ of rest. The slightly lower intensity of $110 \%$ compared with standard $120 \%$ MT intensity was used to accommodate participant tolerability.

\section{Symptom measures}

The participants rated their symptom change on a visual analogue scale (VAS) of $0-100$, in which 0 represented a symptom-free state and 100 represented rocking vertigo so severe that they could not ambulate. Study procedures occurred over a 5-day period with fMRI performed on day 1 and day 5 with the rTMS sessions scheduled in between. The change of the VAS score from day 1 to day 5 was calculated with a minimum change of 10 points on the scale required for significance. The minimum 10-point criterion was chosen because it best correlated with the participants' own perceptions of whether their symptoms had changed. Participants whose symptoms decreased by 10 points or more were considered to be positive responders (improved), those whose scores increased by 10 points or more were considered to be negative responders (worsened), and those with scores in-between were considered to be neutral.

\section{Resting state $\mathrm{fMRI}$ data analysis}

The fMRI data preprocessing was performed using AFNIs (analysis of functional neuroimages). The fMRI data of each subject were spatially coregistered to high-resolution anatomical images, and the first five volumes of each run were excluded from analysis to allow the BOLD signal to reach steady state. Major processing steps included respirationand pulse-associated noise reduction using RETROICOR (Glover et al., 2000), slice timing and rigid-body motion correction, spatial smoothing with a Gaussian kernel (Full Width Half Maximum $=6 \mathrm{~mm}$ ), and temporal filtering with a band-pass filter $(0.005-0.1 \mathrm{~Hz})$. In addition, the low-frequency changes in respiration volume (Birn et al., 2006), six affine motion parameters, signal from a ventricular region of interest (ROI), and signal from a region centered in the white matter were regressed out from the data set. Data points of excessive motion (root mean square of displacement in three dimensions is larger than $0.3 \mathrm{~mm}$ ) were excluded from regression and correlation analysis using the censoring option were implemented in AFNIs (afni_proc.py). Specifically, the L2-norm of motion parameters estimated from motion registration was calculated per run and those time points of amplitude of displacement in three dimensions is larger than $0.3 \mathrm{~mm}$ were censored/ excluded in the regression or in the later calculation of connectivity. Notably the band-pass filtering was implemented in a linear regression manner. Therefore, censoring out points of extraordinary motion did not lead to unusual edge effects as seen in filtering using a convolution form. The whole-brain global signal was not removed as this may lead to spurious anticorrelation (Fox et al., 2009; Murphy et al., 2009). 
RSFC was computed as Pearson's correlation with regard to a seed region (Biswal et al., 1995). Two anatomical regions were investigated, the EC and the stimulated site within the DLPFC, both identified in individuals' anatomical images. We only examined the RSFC seeded in the IEC or rEC. Coordinates of individual EC locations were manually determined on high-solution structural MR images acquired in the coronal plane using anatomical guidelines by Insausti and associates (1998). The seed region of EC was a 2.5-mmradius sphere centered at the $\mathrm{IEC}$ and $\mathrm{rEC}$ in each individual's anatomical space, medial to the collateral sulcus, and lateral to the amygdala in the coronal plane. Each ROI was examined to ensure that none of the EC seeds contained any voxels overlapping with the subarachnoid space. Coordinates for the ROIs for each DLPFC (right and left) that were stimulated were determined in individuals' anatomical space according to the Localite navigation system used during treatment. The DLPFC ROIs were a 7-mm-radii centered in the anterior portion of the middle of the middle frontal gyrus for each hemisphere. DLPFC ROIs were further masked in conjunction with the cortical areas. Correlation maps were produced by extracting the preprocessed BOLD time courses from the seed region, averaging the signals from within the seed region, then computing the correlation coefficients between the seed time course and the time course from all other brain voxels. Correlation maps were computed in single-subject-based space and then converted to normalized space for group-level analysis.

For statistical tests and group analyses, correlation coefficients were converted to a normal distribution by Fisher's $\mathrm{z}$ transform. These values were converted to $\mathrm{z}$ scores (i.e., zero mean, unit variance, Gaussian distributions) by dividing the Fisher-transformed correlation coefficient by the square root of the variance, that is, $1 / \operatorname{sqrt}(n-3)$, in which $n$ is the degree of freedom in the measurements. The degrees of freedom were corrected for the temporal dependence across consecutive time points (Fox et al., 2005). Individuals' structural anatomical images were spatially coregistered to the Talairach and Tournoux (1988) template brain with aid of the Advanced Normalization Tools (www.picsl.upenn.edu/ANTS) for spatial normalization. Individual z-score maps were transformed to the normalization space by applying the coregistration transformation and then submitted for comparison and statistical testing.

\section{RSFC correlation with symptom response}

We next determined whether there is a correlation between clinical response and whole-brain functional connectivity changes to the EC. Specifically, the individual pre-TMS z-score maps were subtracted from the post-TMS $\mathrm{z}$-score maps and whole-brain analysis was performed to test whether post-versus-pre connectivity changes differed in accordance with individual symptom changes measured by VAS scores. First, the participants were categorized, based on VAS score changes, into positive (reduced at least 10 points), neutral (less than 10 points), and negative groups (increased at least 10 points). This categorization was based on the consideration that the VAS scores are subjective reflections of participants' symptoms and their values may not follow a Gaussian distribution. Thus, the connectivity changes were compared according to their categorized responses (positive: 1 , neutral: 0 , and negative: -1 ) in a general linear model, instead of being compared with the VAS values as a continuous variable. The significance criterion for detecting activation was set at $p_{\text {corrected }}<0.05$, which was determined using the AFNI program 3dClustSim (cluster size $>30$ voxels, thresholded at the voxel level at $p<0.005)$. Positive activation indicated that effective reduction of symptoms is associated with a decrease of RSFC and that exacerbation of symptoms is associated with an increase of RSFC. Individual connectivity scores were also extracted from the activation regions and averaged according to their response categorization.

\section{RSFC prediction of treatment response}

Considering that our current stimulation protocol targeted DLPFC, our next analysis was to determine whether the RSFC between the stimulated sites and the EC could predict treatment response. Thus, in the prediction analyses, we examined only the RSFC in the pretreatment baseline data. Our rationale was that the therapeutic effect induced by stimulation might modify the functional connectivity between DLFPC and the EC, given prior connectivity and metabolic differences between these areas in individuals with MdDS compared with controls (Cha et al., 2012). Thus, we examined whether the pretreatment connectivity between DLPFC and the EC was related to the responses among all participants. DLPFC targets were identified in individual anatomical space on the first day of treatment and were consistently used for repeated stimulation for all 5 days. With seeds in the IEC and rEC, the connectivity with the ipsilateral DLPFC ROIs was averaged. Since study participants received bilateral TMS (inhibitory stimulation of the rDLPFC and excitatory stimulation at the IDLPFC) with the goal of manipulating DLPFC-EC connectivity (by allowing release of interhemispheric inhibition from the ipsilateral side), the separate unilateral RSFC measurements between DLPFC and EC (rDLPFC-rEC, IDLPFCIEC, rDLPFC-1EC, and IDLPFC-rEC) were explored. The DLPFC-EC connectivity measurements from the pre-TMS data were compared with the symptom responses using linear regression. Furthermore, we tested whether in any of the responder groups, the pre-TMS baseline connectivity was significantly different from 0 .

\section{Results}

Twenty individuals completed the study with no dropouts. Although headache was the most common side effect reported, no subject had to stop stimulation because of headache. One subject who had a history of migraine headaches developed a migraine headache after one session but was able to avoid further headaches by taking prophylactic ibuprofen. Changes in the magnitude of rocking dizziness from day 1 to day 5 are depicted in Figure 1. Among the 20 participants, 6 improved by 10 points or more (positive responders), 6 worsened by 10 points or more (negative responders), and 8 remained unchanged (neutral responders). Although the overall magnitude of change in symptoms was greater for improvement than for worsening, for the purposes of analyses, we treated the data as categorical variables in subsequent regression analyses. 


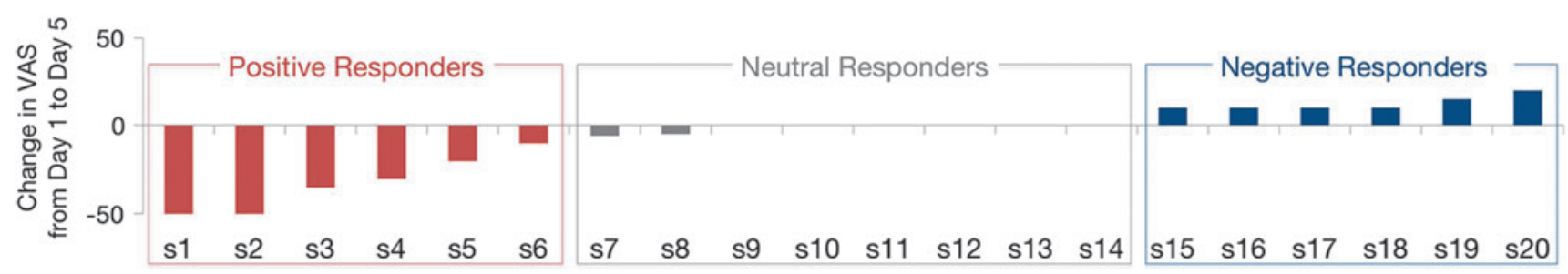

FIG. 1. VAS changes from day 1 to day 5 of treatment. Spread of responses to rTMS on a 0-100 VAS. Subjects S1-S6 represent positive responders whose symptoms decreased with rTMS; S7-S14 showed no response; S15-S20 worsened with treatment. rTMS, repetitive transcranial magnetic stimulation; VAS, visual analogue scale. Color images available online at www.liebertpub.com/brain

We further tested the hypothesis of whether the postversus-pre connectivity changes were related to the symptom outcomes. Whole-brain analysis of RSFC to the lEC showed that connectivity between this region and the $\mathrm{rEC}$, the right inferior parietal lobule, and the left precuneus was significantly modulated by rTMS over DLPFC (Fig. 2). These regions comprise the posterior default mode network.

To determine the directionality of the effects of rTMS on RSFC, we extracted the connectivity scores in the activation regions (Fig. 2), and examined the connectivity changes averaged by response categorization. Specifically, individuals who responded positively to treatment and had symptom reduction exhibited a decrease in functional connectivity between these nodes and the EC, whereas those with no response or worsened symptoms showed an increase in connectivity, showing a directional relationship between functional connectivity and symptoms (Fig. 3). In the region of the right inferior parietal lobule, the group of positive responders showed a significant decrease of connectivity with the IEC ( $p=0.04$, two-sided $t$ test). However, a signif- icant increase in connectivity was observed in the group of negative ( $p=0.007$, two-sided $t$ test) and neutral responders ( $p=0.02$, two-sided $t$ test) with the precuneus.

Pre-rTMS connectivity was then examined to determine how the baseline connectivity between the DLPFC targets and the EC could predict treatment response after rTMS. Figure 4A shows the extent of overlap of the DLPFC ROIs after alignment of all individual DLPFCs into normalized space. Note that there only exists a moderate level of overlap among individuals once the DLPFC was transformed into the standard space. Functional connectivity between DLPFC and EC was calculated from individual ROIs for each region. Baseline functional connectivity between $\mathrm{rDLPFC}$ and $\mathrm{rEC}$ $(\mathrm{r}=-0.65, p=0.002)$ as well as 1DLPFC and 1EC $(\mathrm{r}=-0.57$, $p=0.008$ ) was found to be significantly correlated with symptom reductions after rTMS as shown in Figure 4B. Other unilateral connectivities between DLPFC and EC (IDLPFC-rEC, rDLPFC-lEC) were examined separately and all showed a significant negative correlation with symptom changes after rTMS (all $p<0.01, \mathrm{r} \leq-0.57$,
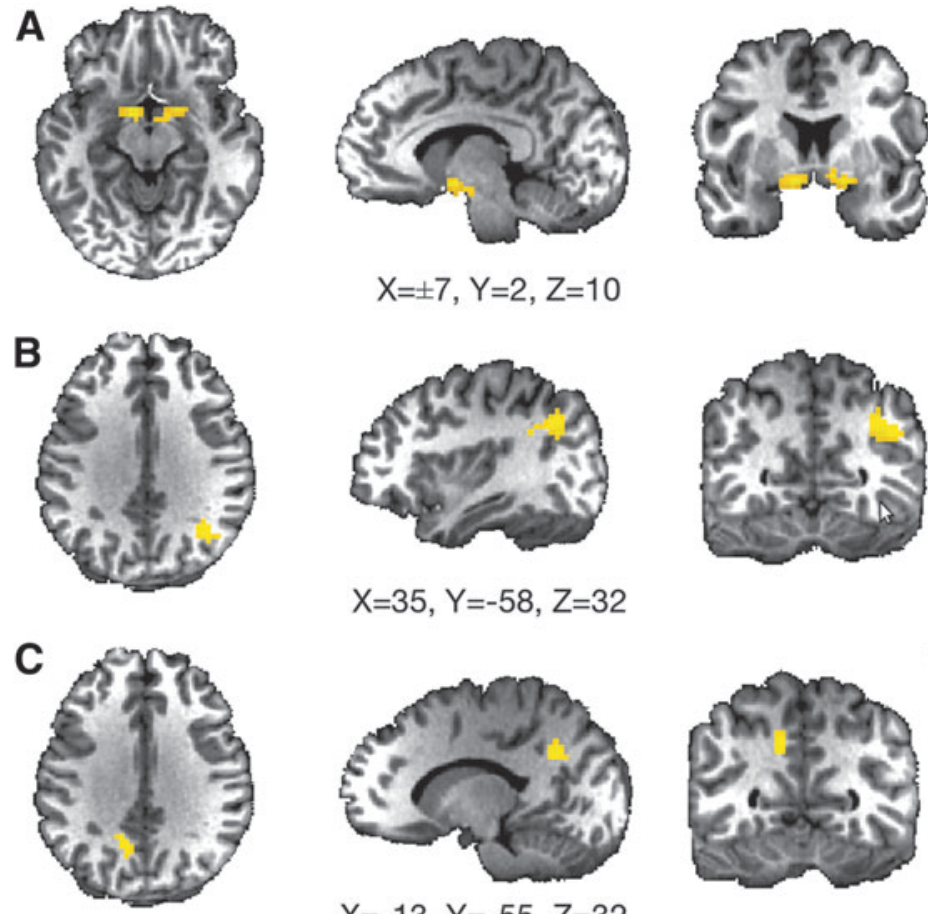

$$
X= \pm 7, Y=2, Z=10
$$

$X=35, Y=-58, Z=32$

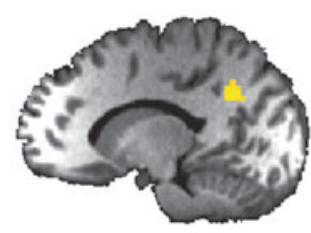

$X=-13, Y=-55, Z=32$
10
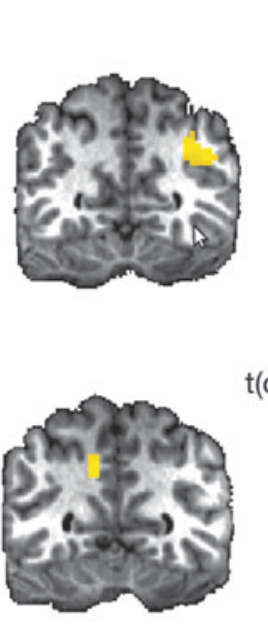

FIG. 2. Connectivity changes before and after rTMS are related to symptom changes in MdDS patients. Whole-brain analysis showing brain areas in which functional connectivity to the $1 \mathrm{EC}$ changes as a function of symptom change after rTMS. The main dynamic areas are (A) the rEC, (B) the right inferior parietal lobule, and $(\mathbf{C})$ the left precuneus. All are components of the posterior default mode network. lEC, left entorhinal cortex; MdDS, mal de debarquement syndrome; rEC, right entorhinal cortex. Color images available online at www.liebertpub .com/brain 
A
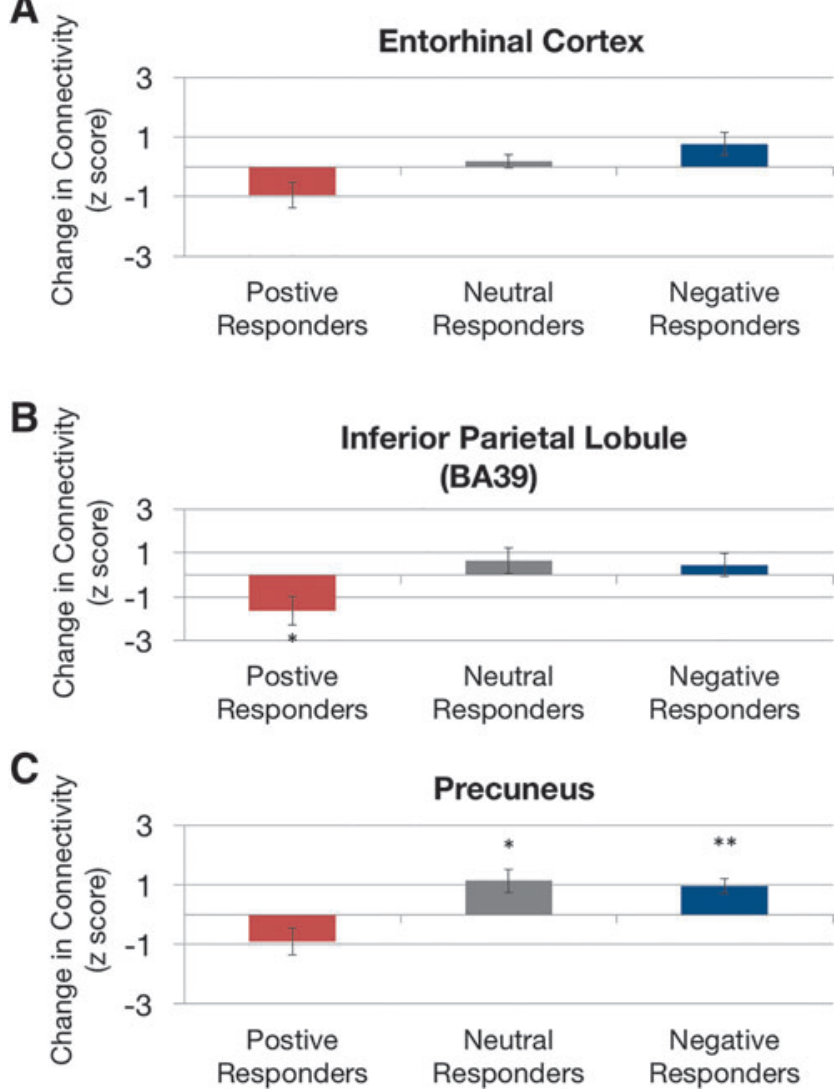

FIG. 3. Directional effect of functional connectivity changes relative to symptom change. Within regions that showed dynamic functional connectivity changes with the IEC [(A) rEC; (B) right inferior parietal lobule, and (C) left precuneus], functional connectivity uniformly decreased in positive responders compared with nonresponders. $*$ and $* *$ indicate significant changes for $p<0.05$ and $p<0.01$, respectively. Color images available online at www.liebertpub.com/brain
Supplementary Fig. S1; Supplementary Data are available online at www.liebertpub.com/brain).

Furthermore, we tested whether in any of the responder group the pre-TMS baseline connectivity was significantly different from 0 . Our results indicated that only in the group of positive responders was baseline connectivity found to be significantly positive (two-sided $t$ test: $p=0.002$ for the rDLPFC-rEC connectivity, $p=0.002$ for the IDLPFC-lEC connectivity, $p=0.02$ for $1 \mathrm{DPFC}-\mathrm{rEC}$ connectivity, but $p>0.05$ for rDPFC-1EC connectivity), indicating that positive (higher) baseline functional connectivity was associated with effective treatment.

\section{Discussion}

Our study has shown that noninvasive brain stimulation with rTMS over DLPFC can be used as a therapeutic tool to alter the perception of self-motion in MdDS. This intervention yielded therapeutic changes that have an RSFC signature. Specifically, improvement in symptom severity was correlated with a reduction in RSFC between the posterior default mode network regions and the EC, an area previously determined to be hypermetabolic in MdDS (Cha et al., 2012). In addition, treatment responses could be predicted to some degree by baseline RSFC between the stimulated area and the EC.

The protocol implemented in the study combined both $1 \mathrm{~Hz}$ rDLPFC and $10 \mathrm{~Hz}$ IDLPFC stimulation with an initial expectation that clinical improvement would be attributed to the DLPFC-EC-RSFC. Both protocols had been used separately in pilot studies with both showing the potential to induce therapeutic responses (Cha et al., 2013, 2016). As left prefrontal EC RSFC was shown to be lower in MdDS than in healthy controls (Cha et al., 2012), we tested the hypothesis that DLPFC stimulation might normalize any baseline connectivity deficits. Instead, we determined that DLPFC stimulation did not lead to increased connectivity between
FIG. 4. Correlation between baseline EC-DLPFC functional connectivity and treatment response. (A) Region of interests over the IDLPFC and rDLPFCs represented in red for left and blue for right. (B) Higher baseline functional connectivity is associated with a greater magnitude of symptom reduction after $\mathrm{rTMS}$, exhibited by both rDLPFC and rEC connectivity in (B1) and 1DLPFC and IEC connectivity in (B2). DLPFC, dorsolateral prefrontal cortex; IDLPFC, left DLPFC; rDLPFC, right DLPFC. Color images available online at www.liebertpub.com/brain

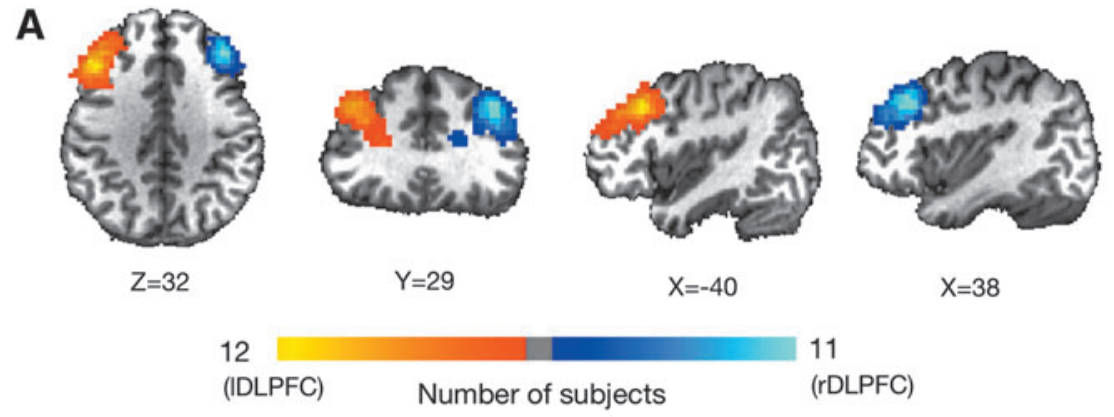

B

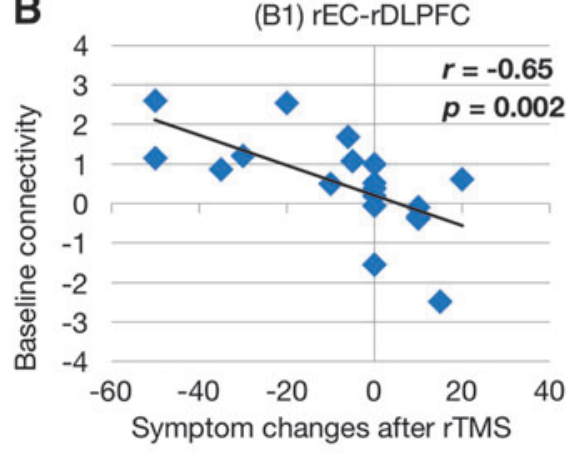

(B2) IEC-IDLPFC

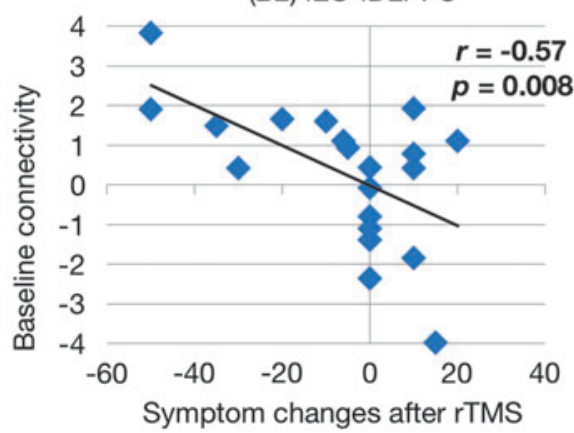


DLPFC and EC, but that DLPFC stimulation lead to decreased connectivity between posterior default mode network and the EC, likely through another pathway. This finding was consistent with our previous data showing that MdDS is also associated with elevated posterior parietal and occipital RSFC to EC compared with healthy controls (Cha et al., 2012), suggesting that rTMS intervenes by normalizing the maladaptive hyperconnectivity.

The fMRI connectivity results of our current investigation are related to our previous investigations using EEG, which both reported a reduction of synchrony in posterior occipital and parietal areas (Ding et.al 2014). In our previous study, a decrease in post-rTMS synchrony (i.e., reduction in ICPC) in the theta and alpha frequency bands was observed as a function of symptom improvement (Ding et al., 2014). Nodes with reduced synchrony (as reflected by EEG) resided over the precuneus and correlated with our finding of precuneusEC functional connectivity reductions related to symptom improvement. The finding that TMS modulates connectivity between the target site and distal areas is corroborated in other studies that used EEG (Chen et al., 2003; Oliviero et al., 2003). The coupled changes of EEG synchrony and fMRI network are also consistent with the findings of direct coupling relationship between resting state EEG and BOLD fMRI signals (Mantini et al., 2007; Yuan et al., 2012, 2013).

The EC, as part of the hippocampal formation, has been recognized as an integral part of the default mode network. In the resting state, the spontaneous activity of entorhinal cortical neurons is coherently connected to other core regions within the default mode network, that is, posterior cingulate gyrus and precuneus, the inferior parietal lobule, ventral medial prefrontal cortex, dorsal medial prefrontal cortex, and lateral temporal cortex (Valenti and Grace, 2009; Van Hoesen et al., 1972). The EC has been shown to generate sustained activity in the absence of continuous neocortical input, making it a biologically plausible nidus for the generation of persistent abnormal motion perception (Dupret and Csicsvari, 2012; Hahn et al., 2012). The medial EC is spatially tuned and receives information from large regions of the neocortex, particularly the somatosensory association cortex, and is the main gateway of neocortical information entering the hippocampus (Coutureau and Di Scala, 2009; Jones and Powell, 1970; Van Hoesen et al., 1972).

Our study is related to other rTMS studies for the indication of major depression, since we employed a common DLPFC stimulation protocol used in an off-label manner. Recent neuroimaging studies suggest that the neuromodulatory effect of rTMS is related to the manipulation of RSFC, which characterizes the resting state synchrony between two discrete regions. The RSFC in response to focal rTMS stimulation has been found in a largely distributed manner. For example, in a population of depressed individuals studied by Liston and associates (2014), stronger clinical responses (reduction of symptoms) were found to be correlated with a reduction of connectivity between the subgenual anterior cingulate cortex (sgACC) and the default mode network. The RSFC before rTMS treatment was found to be abnormally elevated between the sgACC and the default mode network. Interestingly, patients with higher baseline connectivity between sgACC and the default mode network were also those stronger responders to rTMS. Thus, it was suggested that the therapeutic mechanism of rTMS was to effectively normalize functional connectivity. The finding that TMS acts by modulating functional connectivity within cortical networks is corroborated by studies employing rTMS in depression (Kozel et al., 2011), essential tremor (Popa et al., 2013), stroke (Volz et al., 2016), and other behavior modification (Li et al., 2017). Our findings are consistent with these studies such that in MdDS, reduction of symptoms after rTMS treatment was associated with a decrease of RSFC, particularly between the EC and the default mode network.

In addition, we found that baseline RSFC is an important predictor of rTMS response, as higher baseline connectivity was found to predict subsequent clinical improvement. Nevertheless, the nature of connectivity patterns pertinent to the rTMS stimulation site in general has been elusive, ranging from positive correlations to negative correlations. In the study by Liston and associates (2014), positive baseline connectivity between sgACC and a central executive network (containing the stimulation site) was found to be predictive of effective treatment. In biotyping depression according to functional connectivity patterns, individuals with higher RSFC between dorsomedial prefrontal cortex, amygdala, DLPFC, bilateral orbitofrontal cortex, and posterior cingulate cortex responded significantly better to rTMS $(82.5 \%$ vs. $25 \%$ ) than those with lower connectivity (Downar et al., 2014; Drysdale et al., 2017).

However, another study by Fox and associates (2012b) examined the RSFC with respect to direct DLPFC targets in different targeting schemes that were previously reported to reflect differences in clinical efficacy. The connectivity results revealed that DLPFC rTMS sites with better clinical efficacy were more negatively correlated (anticorrelated) with the subgenual cingulate. In addition, even though the IDLPFC exhibits rich connections to many cortical and subcortical regions, including positive correlations with several key regions of the default model network (Fox et al., 2012b), baseline connectivity with regard to the DLPFC simulation site alone is not predictive of treatment (Liston et al., 2014).

In a similar manner, our current study also investigated whether the pre-TMS baseline RSFC was related to clinical response. A significant predictive effect was found for the pre-rTMS RSFC across individuals. Interestingly, the results were consistent with depression studies in showing that baseline RSFC may predict rTMS treatment response. In Liston and associates (2014), positive, higher baseline connectivity between the rTMS-targeted area (IDLPFC) and the intended network (default mode network) was associated with stronger responders. Similarly, in our study, positive baseline connectivity between the rTMS targets (DLPFC in the off-label usage) and the intended target (EC, also considered part of the default model network) was found to be associated with a positive response, that is, greater presimulation connectivity was associated with greater responses.

A limitation to our study is that we did not have a sham rTMS arm of the study. However, by using response to rTMS as a categorical variable, we were able to use nonresponders and individuals who worsened after rTMS as a control group to determine whether there was a directional relationship between symptom change and RSFC, that is, if symptoms went up with one functional change, it should go down with the opposite change. We determined that individuals whose symptoms improved had a greater magnitude 
of symptom change than individuals who showed no effect, however. Moreover, the functional connectivity patterns in the responder group were distinct from those in the neutral and negative responder groups in that they had significantly higher baseline connectivity between DLPFC and the EC than in either of the other groups.

MdDS is a natural model of environmental entrainment effects on the human brain, which by all measures to date have been consistent with an oversynchronization of neural networks. That a chronic symptom can be switched off with noninvasive brain stimulation, and often with very limited amounts of exposure, is support for aberrant functional connectivity, itself being the basis of clinical symptoms. Our program of study has used both EEG and fMRI RSFC biomarkers to both measure and predict treatment response. We hope that this study will contribute to the growing body of literature that supports the use of these functional neuroimaging tools to enhance treatment of human functional brain disorders. Our recent work (Yuan et al., 2016) has shown that electrophysiological networks, including the default mode node work, can be reconstructed from EEG data alone, which also resemble those fMRI network counterparts in terms of spatial patterns as well as temporal dynamics. The capability of imaging resting state network connectivity in multimodal data opens new avenues of integrating neuroimaging and neuromodulation.

\section{Conclusion}

This study shows that persistent feelings of self-motion triggered by entrainment to an external oscillating environment causing the clinical syndrome of MdDS can be reduced with noninvasive brain stimulation and that symptom reduction correlates with functional connectivity reductions between the stimulated site and the EC. Our investigation revealed that individuals with symptom improvement to rTMS exhibit decreased IEC connectivity with the contralateral EC, as well as with the right inferior parietal lobule and precuneus. We also showed that baseline connectivity between the EC and the DLPFC can predict response to treatment. These data have been used to develop more focused and effective neuromodulation treatments for MdDS.

\section{Acknowledgments}

This work was supported by the Laureate Institute for Brain Research, the William K. Warren Foundation, NIH/ NIDCD R03 DC010451 (Y.H.C.), an equipment grant from the MdDS Balance Disorders Foundation (Y.H.C.), and an award through NSF EPSCoR RII Track-2 \#1539068 (H.Y., L.D., Y.H.C.).

\section{Author Disclosure Statement}

No competing financial interests exist.

\section{References}

Birn RM, Diamond JB, Smith MA, Bandettini PA. 2006. Separating respiratory-variation-related fluctuations from neuronal-activity-related fluctuations in fMRI. Neuroimage 31:1536-1548.
Biswal BB, Mennes M, Zuo XN, et al. 2010. Toward discovery science of human brain function. Proc Natl Acad Sci U S A 107:4734-4739.

Biswal BB, Yetkin FZ, Haughton VM, Hyde JS. 1995. Functional connectivity in the motor cortex of resting human brain using echo-planar MRI. Magn Reson Med 34:537-541.

Brown JJ, Baloh RW. 1987. Persistent mal de debarquement syndrome: a motion-induced subjective disorder of balance. Am J Otolaryngol 8:219-222.

Cha YH. 2012. Less common neuro-otologic disorders. Continuum (Minneap Minn) 18:1142-1157.

Cha YH. 2015. Mal de debarquement syndrome: new insights. Ann N Y Acad Sci 1343:63-68.

Cha YH, Brodsky J, Ishiyama G, Sabatti C, Baloh RW. 2008. Clinical features and associated syndromes of mal de debarquement. J Neurol 255:1038-1044.

Cha YH, Chakrapani S. 2015. Voxel based morphometry alterations in mal de debarquement syndrome. PLoS One 10: e0135021.

Cha YH, Chakrapani S, Craig A, Baloh RW. 2012. Metabolic and functional connectivity changes in mal de debarquement syndrome. PLoS One 7:e49560.

Cha YH, Cui Y, Baloh RW. 2013. Repetitive transcranial magnetic stimulation for mal de debarquement syndrome. Otol Neurotol 34:175-179.

Cha YH, Urbano D, Pariseau N. 2016. Randomized single blind sham controlled trial of adjunctive home-based tDCS after rTMS for mal de debarquement syndrome: safety, efficacy, and participant satisfaction assessment. Brain Stimul 9: 537-544.

Chen WH, Mima T, Siebner HR, Oga T, Hara H, Satow T, et al. 2003. Low-frequency rTMS over lateral premotor cortex induces lasting changes in regional activation and functional coupling of cortical motor areas. Clin Neurophysiol 114: 1628-1637.

Coutureau E, Di Scala G. 2009. Entorhinal cortex and cognition. Prog NeuroPsychopharmacol Biol Psychiatry 33: 753-761.

Dickson CT, Magistretti J, Shalinsky M, Hamam B, Alonso A. 2000. Oscillatory activity in entorhinal neurons and circuits. Mechanisms and function. Ann N Y Acad Sci 911:127-150.

Di Lazzaro V, Dileone M, Pilato F, Capone F, Musumeci G, Ranieri F, et al. 2011. Modulation of motor cortex neuronal networks by rTMS: comparison of local and remote effects of six different protocols of stimulation. J Neurophysiol 105: 2150-2156.

Ding L, Shou G, Yuan H, Urbano D, Cha YH. 2014. Lasting modulation effects of rTMS on neural activity and connectivity as revealed by resting state EEG. IEEE Trans Biomed Eng 61:2070-2080.

Downar J, Geraci J, Salomons TV, Dunlop K, Wheeler S, McAndrews MP, et al. 2014. Anhedonia and reward-circuit connectivity distinguish nonresponders from responders to dorsomedial prefrontal repetitive transcranial magnetic stimulation in major depression. Biol Psychiatry 76:176-185.

Drysdale AT, Grosenick L, Downar J, Dunlop K, Mansouri F, Meng Y, et al. 2017. Resting-state connectivity biomarkers define neurophysiological subtypes of depression. Nat Med 23:28-38.

Dupret D, Csicsvari J. 2012. The medial entorhinal cortex keeps Up. Nat Neurosci 15:1471-1472.

Egorov AV, Hamam BN, Fransén E, Hasselmo ME, Alonso AA. 2002. Graded persistent activity in entorhinal cortex neurons. Nature 420:173-178. 
Fox MD, Buckner RL, White MP, Greicius MD, Pascual-Leone A. 2012b. Efficacy of transcranial magnetic stimulation targets for depression is related to intrinsic functional connectivity with the subgenual cingulate. Biol Psychiatry 72:595-603.

Fox MD, Halko MA, Eldaief MC, Pascual-Leone A. 2012a. Measuring and manipulating brain connectivity with resting state functional connectivity magnetic resonance imaging (fcMRI) and transcranial magnetic stimulation (TMS). Neuroimage 62:2232-2243.

Fox MD, Snyder AZ, Vincent JL, Corbetta M, Van Essen DC, Raichle ME. 2005. The human brain is intrinsically organized into dynamic, anticorrelated functional networks. Proc Natl Acad Sci U S A 102:9673-9678.

Fox MD, Zhang D, Snyder AZ, Raichle ME. 2009. The global signal and observed anticorrelated resting state brain networks. J Neurophysiol 101:3270-3283.

George MS, Stallings LE, Speer AM, Nahas Z, Spicer KM, Vincent DJ, Bohning DE, Cheng KT, Molloy M, Teneback CC. 1999. Prefrontal repetitive transcranial magnetic stimulation (rTMS) changes relative perfusion locally and remotely. Hum Psychopharmacol Clin Exp 14:161-170.

George MS, Wassermann EM, Kimbrell TA, Little JT, Williams WE, Danielson AL, et al. 1997. Mood improvement following daily left prefrontal repetitive transcranial magnetic stimulation in patients with depression: a placebo-controlled crossover trial. Am J Psychiatry 154:1752-1756.

George MS, Wassermann EM, Williams WA, Callahan A, Ketter TA, Basser P, et al. 1995. Daily repetitive transcranial magnetic stimulation (rTMS) improves mood in depression. Neuroreport 6:1853-1856.

Glover GH, Li TQ, Ress D. 2000. Image-based method for retrospective correction of physiological motion effects in fMRI: RETROICOR. Magn Reson Med 44:162-167.

Gordon CR, Spitzer O, Doweck I, Melamed Y, Shupak A. 1995. Clinical features of mal de debarquement: adaptation and habituation to sea conditions. J Vestib Res 5:363-369.

Hahn TT, McFarland JM, Berberich S, Sakmann B, Mehta MR. 2012. Spontaneous persistent activity in entorhinal cortex modulates cortico-hippocampal interaction in vivo. Nat Neurosci 15:1531-1538.

Hain TC, Hanna PA, Rheinberger MA. 1999. Mal de debarquement. Arch Otolaryngol Head Neck Surg 125:615-620.

Hallett M. 2007. Transcranial magnetic stimulation: a primer. Neuron 55:187-199.

Hasselmo ME, Fransen E, Dickson C, Alonso AA. 2000. Computational modeling of entorhinal cortex. Ann N Y Acad Sci 911:418-446.

Insausti R, Juottonen K, Soininen H, Insausti AM, Partanen K, Vainio P, et al. 1998. MR volumetric analysis of the human entorhinal, perirhinal, and temporopolar cortices. Am J Neuroradiol 19:659-671.

Irwin JA. 1881. The pathology of sea-sickness. Lancet 118:907909.

Jacob PY, Poucet B, Liberge M, Save E, Sargolini F. 2014. Vestibular control of entorhinal cortex activity in spatial navigation. Front Integr Neurosci 8:38.

Jones EG, Powell TP. 1970. An anatomical study of converging sensory pathways within the cerebral cortex of the monkey. Brain 93:793-820.

Kajiwara R, Takashima I, Mimura Y, Witter MP, Iijima T. 2003. Amygdala input promotes spread of excitatory neural activity from perirhinal cortex to the entorhinal-hippocampal circuit. J Neurophysiol 89:2176-2184.
Knoch D, Treyer V, Regard M, Müri RM, Buck A, Weber B. 2006. Lateralized and frequency-dependent effects of prefrontal rTMS on regional cerebral blood flow. Neuroimage 31:641-648.

Kozel FA, Rao U, Lu H, Nakonezny PA, Grannemann B, McGregor T, et al. 2011. Functional connectivity of brain structures correlates with treatment outcome in major depressive disorder. Front Psychiatry 2:7.

Li X, Du L, Sahlem GL, Badran BW, Henderson S, George MS. 2017. Repetitive transcranial magnetic stimulation (rTMS) of the dorsolateral prefrontal cortex reduces resting-state insula activity and modulates functional connectivity of the orbitofrontal cortex in cigarette smokers. Drug Alcohol Depend 174:98-105.

Li X, Nahas Z, Kozel FA, Anderson B, Bohning DE, George MS. 2004. Acute left prefrontal transcranial magnetic stimulation in depressed patients is associated with immediately increased activity in prefrontal cortical as well as subcortical regions. Biol Psychiatry 55:882-890.

Liston C, Chen AC, Zebley BD, Drysdale AT, Gordon R, Leuchter B, et al. 2014. Default mode network mechanisms of transcranial magnetic stimulation in depression. Biol Psychiatry 76:517-526.

Mantini D, Perrucci MG, Del Gratta C, Romani GL, Corbetta M. 2007. Electrophysiological signatures of resting state networks in the human brain. Proc Natl Acad Sci U S A 104: 13170-13175.

Murphy K, Birn RM, Handwerker DA, Jones TB, Bandettini PA. 2009. The impact of global signal regression on resting state correlations: are anti-correlated networks introduced? Neuroimage 44:893-905.

Noh NA, Fuggetta G, Manganotti P, Fiaschi A. 2012. Long lasting modulation of cortical oscillations after continuous theta burst transcranial magnetic stimulation. PLoS One 7:e35080.

Oliviero A, Strens LHA, Lazzaro V, Tonali PA, Brown P. 2003. Persistent effects of high frequency repetitive TMS on the coupling between motor areas in the human. Exp Brain Res 149:107-113.

Padberg F, George MS. 2009. Repetitive transcranial magnetic stimulation of the prefrontal cortex in depression. Exp Neurol 219:2-13.

Popa T, Russo M, Vidailhet M, Roze E, Lehéricy S, Bonnet C, et al. 2013. Cerebellar rTMS stimulation may induce prolonged clinical benefits in essential tremor, and subjacent changes in functional connectivity: an open label trial. Brain Stimul 6:175-179.

Shou G, Yuan H, Urbano D, Cha YH, Ding L. 2014. Changes of symptom and EEG in mal de debarquement syndrome patients after repetitive transcranial magnetic stimulation over bilateral prefrontal cortex: a pilot study. Conf Proc IEEE Eng Med Biol Soc 2014:4294-4297.

Speer AM, Kimbrell TA, Wassermann EM, D Repella J, Willis MW, Herscovitch P, Post RM. 2000. Opposite effects of high and low frequency rTMS on regional brain activity in depressed patients. Biol Psychiatry 48:1133-1141.

Tahvildari B, Fransén E, Alonso AA, Hasselmo ME. 2007. Switching between "On" and "Off" states of persistent activity in lateral entorhinal layer III neurons. Hippocampus 17:257-263.

Talairach J, Tournoux P. 1998. Co-planar stereotaxic atlas of the human brain. In: 3-Dimensional Proportional System: An Approach to Cerebral Imaging. New York, NY: Thieme Medical Publishers. 
Valenti O, Grace AA. 2009. Entorhinal cortex inhibits medial prefrontal cortex and modulates the activity states of electrophysiologically characterized pyramidal neurons in vivo. Cereb Cortex 19:658-674.

Van Hoesen GW, Pandya DN, Butters N. 1972. Cortical afferents to the entorhinal cortex of the rhesus monkey. Science 175:1471.

Volz LJ, Rehme AK, Michely J, Nettekoven C, Eickhoff SB, Fink GR, Grefkes C. 2016. Shaping early reorganization of neural networks promotes motor function after stroke. Cereb Cortex 26:2882-2894.

Yuan H, Ding L, Zhu M, Zotev V, Phillips R, Bodurka J. 2016. Reconstructing large-scale brain resting-state networks from high-resolution EEG: spatial and temporal comparisons with fMRI. Brain Connect 6:122135.

Yuan H, Young KD, Phillips R, Zotev V, Misaki M, Bodurka J. 2014. Resting-state functional connectivity modulation and sustained changes after real-time functional magnetic reso- nance imaging neurofeedback training in depression. Brain Connect 4:690-701.

Yuan H, Zotev V, Phillips R, Bodurka J. 2013. Correlated slow fluctuations in respiration, EEG, and BOLD fMRI. Neuroimage 79:81-93.

Yuan H, Zotev V, Phillips R, Drevets WC, Bodurka J. 2012. Spatiotemporal dynamics of the brain at rest-exploring EEG microstates as electrophysiological signatures of BOLD resting state networks. Neuroimage 60:2062-2072.

Address correspondence to:

Han Yuan

Stephenson School of Biomedical Engineering

University of Oklahoma

3100 Monitor Avenue Suite 125

Norman, OK 73072

E-mail: hanyuan@ou.edu 